\title{
Calcium phosphate coated core- shell protein nanocarriers: robust stability, controlled release and enhanced anticancer activity for curcumin delivery
}

by Wu, Q., Gao, H., Vriesekoop, F., Liu, Z., He, J. and Liang, $\mathrm{H}$.

Copyright, publisher and additional Information: This is the author accepted manuscript. The final published version (version of record) is available online via Elsevier.

This version is made available under the CC-BY-ND-NC licence: https://creativecommons.org/licenses/by-nc-nd/4.0/legalcode

Please refer to any applicable terms of use of the publisher

DOI: https://doi.org/10.1016/j.msec.2020.111094 


\section{Highlights}

- NaCas@CaP nanodelivery system has nano-size and core-shell structure.

- The mechanism of encapsulation was characterized and analyzed.

- The stability of the encapsulated Cur was significantly improved.

- The pH-responsive release around the cancer cell was achieved.

- The encapsulated Cur existed better cellular anti-oxidant and anti-cancer ability. 
1 Calcium Phosphate Coated Core-Shell Protein

2 Nanocarriers: Robust Stability, Controlled Release

3 and Enhanced Anticancer Activity for Curcumin

4 Delivery

5

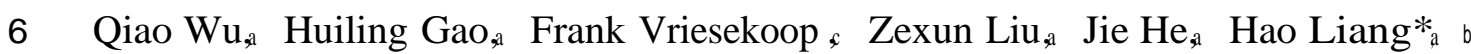

7 a State Key Laboratory of Chemical Resource Engineering, Beijing University of

8 Chemical Technology, Beijing100029, P.R. China

9 b Qinhuangdao Bohai Biological Research Institute of Beijing University of Chemical

10 Technology, Qinhuangdao 066000, China

11 c Department of Food Technology and Innovation, Harper Adams University,

12 Newport TFl0 8NB, Shropshire, United Kingdom

13 Corresponding Author

14 * E-mail: lianghao@ rnail.buct.edu.cn

16 ABSTRACT: Composite protein and inorganic nanodelivery systems can realise a

$17 \mathrm{pH}$-responsive release and effectively improve the stability and anti-cancer

18 proliferative activity of hydrophobic molecules. In this study, a novel core-shell 
21 enhanced stability was built. By using Cur (Curcumin) as a model bioactive molecule,

structure of NaCas (Sodium Caseinate)@ CaP (Calcium Phosphate) as a nanodelivery system with $\mathrm{NaCas}$ as the core for increasing solubility and $\mathrm{CaP}$ as the shell for (Cur@NaCas)@CaP nanoparticles (NPs) demonstrated a uniform size distribution of 150-200 nm with a distinct nano-composite structure. After exposure to $80^{\circ} \mathrm{C}$ for $2 \mathrm{~h}$, the NaCas@CaP loaded Cur still retained 80\% stability while under the same conditions only $12 \%$ of free Cur remained intact. UV-light stability was remarkably enhanced 8.56 fold by the protection of the core-shell structure. More importantly, $\mathrm{pH}$-responsive release was achieved owing to the $\mathrm{CaP}$ surface coating. The encapsulated Cur by NaCas@CaP NPs exhibited an enhanced cellular anti-oxidant activity (CAA) based on MGC-803 cell monolayer models. The confocal laserscanning microscopy (CLSM) images and cancer-cell-proliferation assay illustrated that (Cur@NaCas)@CaP NPs showed significantly improvements of cellular uptake and anti-cancer activity against A549 cancer cells than free Cur. These novel coreshell NaCas@CaP NPs are very promising for intensifying the stability and bioactivity of hydrophobic compounds in drug delivery and cancer treatment.

\section{5}

Key words: Nanodelivery, Sodium caseinate, Calcium phosphate, Curcumin, Antioxidant, Anti-cancer

8 
40 Natural self-assembled delivery systems are nanodelivery systems formed by the

41 self-assembly of natural biomaterials with nanostructures.[1,2] These biomaterials

42 can spontaneously form regular shapes, such as spheres, cylinders, lamellae, etc., by

43 relatively weak non-covalent interactions at concentrations well above their critical

44 micelle concentration.[3-5] These systems are mainly used to encapsulate, protect and

45 transport functional active substances because of their enhanced permeability and

46 retention effect.[6, 7] The enhanced permeability and retention effect refers to the

47 notion that certain macromolecules and nanoparticles accumulate inside tumor tissues

48 at greater concentrations compared to non-tumor tissues.[8] Compared with

49 traditional embedding technologies, natural self-assembled delivery systems have

50 size-related effects, due to the relatively random nature of the self-assembly process

51 which results in a heterogeneous range of surfaces and sized particles. Furthermore,

52 natural self-assembled delivery systems also have different in vivo distribution

53 specificity with regards to the targeted delivery of drugs and nutritional

54 compounds.[9, 10] Moreover, biodegradable self-assembled delivery systems improve

55 the bioavailability of guest molecules, and can achieve a controlled and sustained

56 release, and facilitate targeted delivery of bioactive compounds.[11] Due to their

57 biodegradability, natural self-assembled delivery systems pose minimal cytotoxicity,

58 are renewable and have an abundant availability, a high drug binding capacity, and a

59 significant uptake by target cells, protein-based delivery systems, including zein,[11]

60 soy protein isolate,[12] sodium caseinate[13] and ferritins,[14] have received

61 increasing attention as natural self-assembled delivery systems. 

distinct hydrophobic and hydrophilic domains, NaCas can self-assemble into a stable

64 micellar structure in aqueous solution, especially when its concentration is higher than

65 the its critical micelle concentration.[13] Due to its excellent biocompatibility and 66 biodegradability, and limited immunogenicity, NaCas is regularly employed as a 67 unique natural self-assembled nanodelivery material in biological applications.[15]

68 NaCas has been used to enhance the solubility, stability and bioavailability of 69 otherwise insoluble natural compounds, especially those with considerable biological 70 activity, such as some vitamins and their precursors, [16-18] and antioxidants.[19]

71 Although the deployment of $\mathrm{NaCas}$ is a strategic improvement in the delivery of 72 otherwise recalcitrantly soluble bioactive molecules, the long-term stability of NaCas 73 nanoparticles (NPs) remains a major obstacle for their successful applications as 74 delivery vehicles. The structural integrity of $\mathrm{NaCas}$ is readily compromised as a 75 consequence of changes in $\mathrm{pH}$, ionic strength, water activity, temperature or 76 pressure,[20] which causes an imbalance between hydrophobic and electrostatic 77 interactions.[21, 22] Various strategies for improving the stability of NaCas 78 nanostructures are emerging. Organic materials such as pectin,[23] chitosan,[24] 79 alginates, [25] and combinations thereof [26] have been used to stabilize NaCas NPs. 80 However, even these nanocarriers still fall short of providing sufficient 81 physicochemical stability and often require complicated assembly processes.[27, 28] 82 Therefore, a stable nanoscale NaCas system with a simple assembly method for its 83 synthesis is urgently needed for the delivery of sensitive molecules. 
Hybridization of organic and inorganic carriers can confer nanomaterials with new

85 stability related properties not normally associated with the individual carriers.[29, 30] Calcium phosphate (CaP) NPs have good adsorption capacity and pH-responsive release characterization for both proteins and drugs.[31-33] CaP NPs can deliver diagnostic and therapeutic agents with minimal toxicity for the detection and treatment of various diseases including cancer.[34]In order to improve the stability of NaCas particles, we chose $\mathrm{CaP}$ to provide a secondary coating and as such address the premature release from compounds encapsulated by NaCas.[13] We constructed hybrid NaCas-CaP NPs (NaCas@CaP NPs) with a stable core-shell structure by means of a simple, one-pot method under mild conditions using curcumin (Cur), a natural anti-cancer biomolecule, as a model guest molecule (Scheme 1). Cur was encapsulated into the core of $\mathrm{NaCas}$ micellar particles by hydrophobicinteractions during the self-assembly process of dissociated NaCas, yielding Cur@NaCas NPs.

97 The Cur@NaCas surface was simultaneously coated with $\mathrm{CaP}$ as a stable shell. The 98 formation and conformation of the encapsulated-core-and-shell NPs were 99 characterized by various means. The stability and in vitro releasing of 100 (Cur@NaCas)@CaP NPs at physiological pH and the pH encountered in cancer cells 101 were examined. In addition, the cellular uptake ability, the cellular anti-oxidant 102 activity and in vitro cell proliferation assay of the inclusion complexes were tested 103 and compared with free Cur. Our results indicated that the NaCas@CaP NPs with 104 high efficiency and stability are a novel nanoplatform for cancer therapy and free 
radical scavenging in vivo. Furthermore, the NPs have universality for hydrophobic sensitive molecules, which make these NPs a promising drug delivery system.

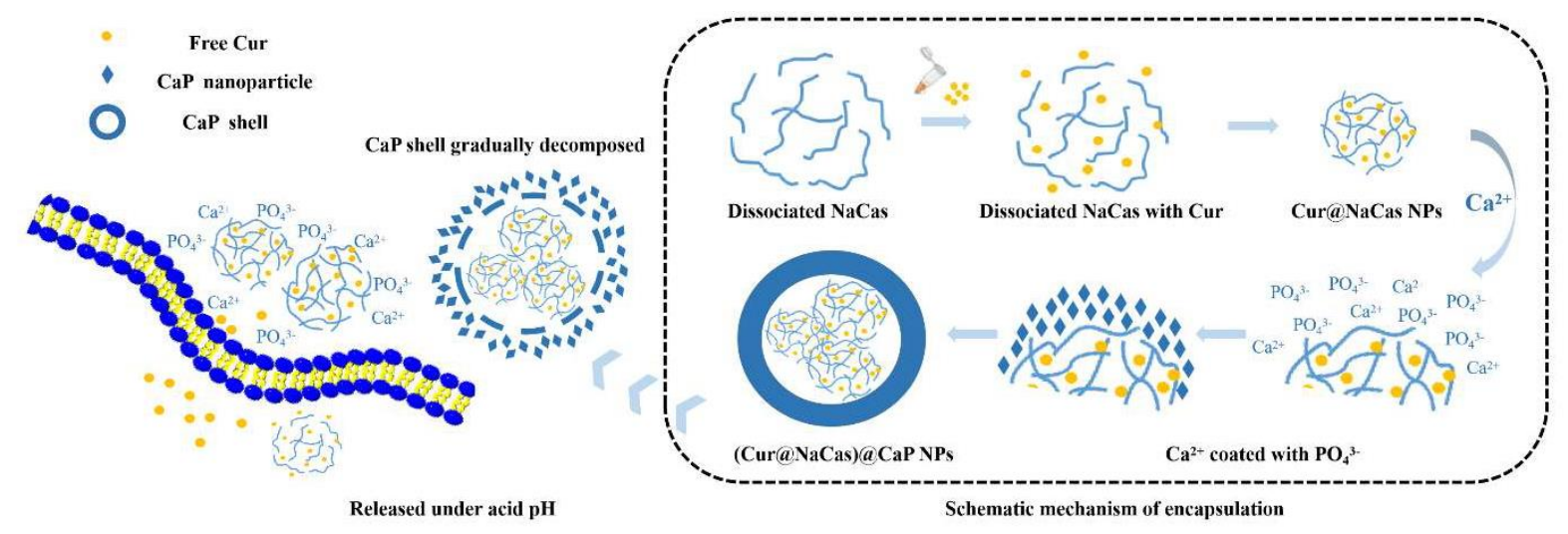

Scheme 1 Schematic mechanism of encapsulation and cell-uptake of NaCas@CaP loaded Cur.

\section{Material and methods}

\subsection{Materials and Cell Culture}

Curcumin (Cur, $98 \%$ ), sodium caseinate (NaCas) were purchased from Macklin Biochemical Co., Ltd, (Shanghai, China). 2,2-azobis (2-amidinopropane) dihydrochloride (ABAP), 2,7-dichlorodi-hydrofluorescein diacetate (DCFH-DA), and Propidium Iodide (PI) were obtained from Sigma-Aldrich, (St Louis, MO, USA). Cell Counting Kit-8 (CCK-8) was obtained from Dojindo Laboratories (Tokyo, Japan). All other chemicals (analytical grade) were purchased from Beijing Chemical Works (Beijing, China), and were applied without further purification. Human gastric cancer cells (MGC803) and human alveolar basal epithelial cancer cells (A549 cells) were obtained from the Cell Resource Center, Peking Union Medical College (Beijing, 
123 China), and cultured with DMEM supplemented with $10 \%$ Fetal Bovine Serum and

$1241 \% \mathrm{P} / \mathrm{S}$ at $95+\% \mathrm{RH}$ with $5 \% \mathrm{CO}_{2}$ at $37^{\circ} \mathrm{C}$.

\section{$125 \quad 2.2$ Preparation of (Cur@NaCas)@CaP NPs}

126 Samples were prepared by rehydrating NaCas in $10 \mathrm{mM}$ phosphate buffer solution

127 ( $\mathrm{pH} 7.4)$ at room temperature $\left(25^{\circ} \mathrm{C}\right)$ with moderate stirring for 30 min to produce a

128 NaCas stock solution at $1 \mathrm{mg} / \mathrm{mL}$. A Cur stock solution of $500 \mu \mathrm{g} / \mathrm{mL}$ in absolute

129 ethyl alcohol was prepared. $200 \mu \mathrm{L}$ of the Cur stock solution was vigorously mixed at

$130600 \mathrm{rpm}$ with $1 \mathrm{~mL} \mathrm{NaCas}$ solution at room temperature for $2 \mathrm{~h}$. The successfully

131 encapsulated Cur (Cur@NaCas) was separated from the non-encapsulated Cur by

132 centrifugation at $290 \mathrm{~g}$ for $10 \mathrm{~min}$. The resultant supernatant containing the

133 Cur@NaCas was subjected to (i) secondary encapsulation, (ii) particle property

134 characterization or (iii) freeze-drying to produce Cur@NaCas NPs for further study.

135 Un-encapsulated Cur on the bottom was measured in order to calculate the drug

136 loading efficiency (DLE). Free curcumin was determined by UV-vis at $428 \mathrm{~nm}$. The

137 DLE of Cur@NaCas was calculated according to following eq.(1):

( $\operatorname{DLE}(\%)=\frac{\text { total amount of added Cur }- \text { amount of Cur on the bottom }}{\text { total amount of added Cur }} \times 100 \%$

139 To facilitate the secondary encapsulation, $20 \mu \mathrm{L}$ calcium chloride $(\mathrm{CaCb})$ solution

140 (50 mM) was added to the Cur@ NaCas NPs solution in PBS (10 mM, pH 7.4) to form

141 (Cur@NaCas)@CaP NPs. The mixture was mixed using a vortex mixer (SCILOGEX

$142 \mathrm{MX}-\mathrm{S}$ ) for $2 \mathrm{~min}$ and then allowed to stand for $24 \mathrm{~h}$. The precipitate was either used

143 for immediate particle property characterization or it was freeze-dried to produce the 
144 (Cur@NaCas)@CaP NPs powders that were used for further study. Un-encapsulated

145 Cur@NaCas on the supernatant was measured in order to calculate the DLE. The

146 DLE of (Cur@NaCas)@CaP was calculated according to following eq.(2).

(2) DLE $=\frac{\text { total amount of added Cur@NaCas - amount of Cur@ NaCas in supernatant }}{\text { total amount of added Cur@ NaCas }} \times 1000,0$

\subsection{Characterization}

149 The various samples used and constructed in this study (NaCas, Cur@ NaCas and

150 (Cur@NaCas)@CaP) were investigated using transmission emission microscopy

151 (TEM, Hitachi HT7700, Tokyo, Japan) and scanning electron microscope (SEM,

152 Hitachi S-4700, Tokyo, Japan).[35] The average size and zeta-potential of free Cur

153 and the various inclusion complexes were ascertained using dynamic light scattering

154 (DLS, Nano-ZS 2000, Malvern Instruments, UK). The crystalline characterization of 155 free Cur and the various inclusion complexes was facilitated using X-ray 156 diffractometer (XRD, D8 ADVANCE, Germany, Bruker) at a diffraction angle of 20 157 ranging from $5^{\circ}$ to $50^{\circ}$ with a stepwise scan rate of $10^{\circ} / \mathrm{min}$ at $0.2^{\circ}$ per step. The FT158 IR spectra of freeze-dried samples were obtained using a fourier-transform infrared 159 spectrometer (FT-IR, Nicolet 6700, Madison, WI, USA) with a scanning range of $1604000-500 \mathrm{~cm}-.^{1}$ The fluorescence spectra for free Cur and encapsulated Cur were 161 recorded by employing a fluorescence spectrophotometer (Hitachi F7000, Tokyo, 162 Japan) at an excitation wavelength of $420 \mathrm{~nm}$, with the emission spectra being 163 recorded of a range from $440-650 \mathrm{~nm}$ at a slit width of $5 \mathrm{~nm}$. Dispersions of 164 inclusion complexes were prepared in deionized water. Free Cur was dissolved in 165 ethanol or deionized water to the same final concentration as in the encapsulated Cur.

\subsection{In vitro stability of nano-encapsulated Cur}

167 The in vitro stability of free Cur and the various NPs was determined according to a previously reported method.[12] Following the various treatments, the samples were 
centrifuged and the ethanolic supernatant was analyzed by HPLC(Liquid column: $\mathrm{C}_{18}$ (200 mmx $4.6 \mathrm{~mm}, 5 \mu \mathrm{m}$ ); Mobile phase: 0.1\% phosphate - acetonitrile (50:50); Detection wavelength: $430 \mathrm{~nm}$; Velocity of flow: $1.0 \mathrm{ml} / \mathrm{min} ; 25^{\circ} \mathrm{C}$; Injection volume: $20 \mu \mathrm{L})$. The initial data for all the cases was set as $100 \%$.

\subsection{In vitro release of Cur}

The in vitro release of Cur from NaCas or NaCas@CaP NPs was established by a dialysis protocol according to a method reported elsewhere.[36] First, Cur loaded inclusion complex were fully dispersed in a release medium (PBS, $\mathrm{pH} 7.4$ or $\mathrm{pH}$ 5.5) in a dialysis bag (MWCO 3500Da), which was then put into a dissolution apparatus, and immersed in $200 \mathrm{~mL}$ of the dissolution medium with $13 / 4(\mathrm{w} / \mathrm{v})$ Tween-80 under gentle stirring at a rate of $100 \mathrm{rpm}$ at $37^{\circ} \mathrm{C}$. At various time intervals, an aliquot of the sample was extracted and analyzed to determine the release of Cur by HPLC.

\subsection{Cellular Uptake Measured by Confocal Laser Scanning Microscope.}

A549 cells $\left(4 \times 10^{4}\right.$ cells/well $)$ were attached to a confocal laser dish over a $12 \mathrm{~h}$ period, after which the cells were washed once with PBS and treated with either free Cur or Cur-loaded NPs $\left(2 \mathrm{~mL}, 5 \mu \mathrm{g} / \mathrm{mL}\right.$, in DMEM) and incubated at $37^{\circ} \mathrm{C}, 5 \% \mathrm{CO}_{2}$ for $6 \mathrm{~h}$. Then the cells were washed twice with PBS to remove all extracellular Cur. A $4 \%$ paraformaldehyde solution was added to fix the cells (at room temperature for 30 min), after which $200 \mu \mathrm{L}$ PI was added to stain the cell nucleus for $15 \mathrm{~min}$. Prior to imaging, all the cells samples were washed once more with PBS. The images of confocal laser scanning microscope (CLSM) were captured by exciting Cur at 442 
run, PI at 488 run, with emissions measured at 475 run and 630 run respectively.

191

192

\subsection{Cellular Antioxidant Activity}

Cellular antioxidant activity (CAA)was determined according to the method reported previously.[35]Briefly, MGC803 cells were seeded in 96-well plates with a concentration of $2.5 \times 10^{5}$ cells per milliliter and incubated for $24 \mathrm{~h}$. Then the growth medium was removed and replaced with either $100 \mu \mathrm{L}$ fresh medium containing 2 $\mu \mathrm{g} / \mathrm{mL}$ free Cur or Cur-loaded NPs and incubated for another $24 \mathrm{~h}$. After 24h, fresh medium $(100 \mu \mathrm{L})$ containing $25 \mu \mathrm{M}$ DCFH-DA was added into each well and incubated for $1 \mathrm{~h}$. Then, all wells were rinsed with PBS before $100 \mu \mathrm{L}$ fresh medium containing $600 \mu \mathrm{M}$ ABAP was added into each well. A microplate reader employing an excitation wavelength of 485 run and an emission wavelength of 535 run was used to measure the fluorescent intensity of samples, over an $1 \mathrm{~h}$ period at 5 minutes intervals. The control samples were treated with DCFH-DA and ABAP only, while the blank samples contained cells treated with DCFH-DA only. The area under the time-fluorescence intensity curve can be obtained by integration, and the antioxidant activity (CAA) units for natural antioxidant can be calculated using the following eq.(3):

(3) $\mathrm{CAA}=100-\left(f_{S A} / f_{C A}\right) \times 100$

f $S A$ represent the integral area of the fluorescence value-time curve by adding different concentrations of Cur;

$f_{C A}$ represent the integral area of the blank group fluorescence value-time curve. 
A549 cells were seeded into 96-well microtiter plates at a density of 4000 cells per

213 well in $100 \mu \mathrm{L}$ of medium. After $24 \mathrm{~h}$, the cells were treated with a medium

214 containing DMSO-dissolved Cur, NaCas-encapsulated or NaCas@CaP-encapsulated

215 Cur. Other cells were untreated (negative controls) or treated only with DMSO,

$216 \mathrm{NaCas}$ or NaCas@CaP NPs at the concentrations as in the dispersions with

217 encapsulated curcumin (positive controls). After $72 \mathrm{~h}$ treatmen,t CCK-8 solution (20

$218 \mu \mathrm{L}$ ) was added to each well and then incubated for $1 \mathrm{~h}$. The absorbance at $450 \mathrm{~nm}$ was 219 measured with a microplate reader (Bio-Tek Instruments, Winooski, VT). The

220 normalized cell viability was obtained after normalizing the viability of a treatment by

221 the viability of control cells treated with DMSO only. The cell viability can be 222 calculated using the following eq.(4):

(4) Cell viability $=($ Atreated/AcontroD X $100 \%$

224 where Atreated and Acontrol are the absorbance of the wells with cells treated by Cur and 225 the control, respectively. The mean and standard deviation from three-well replicates 226 were calculated.

\section{Results and discussion}

\subsection{Preparation and Characterization of CaP coated Core-Shell nanoparticles}

230 (Cur@NaCas)@CaP NPs were prepared using the self-assembly and surface-

231 coating method outlined above. By adjusting the concentration of NaCas and the 232 stirring speed during the encapsulation access, the optimized conditions were 

achieving a DLE of $90.01 \pm 1.29 \%$. See the supplementary data for more in-depth data 235 about the optimization of this methodology.

236 After that, calcium ions as $\mathrm{CaCh}$ were added which interacted with the phosphate 237 in PBS, which in tum allowed the formation of a CaP coating on the surface ofNaCas 238 protein to obtain Cur@NaCas)@CaP. The DLE of (Cur@NaCas)@CaP under 239 optimized conditions of CaP (Table S4) was 99.25 $\pm 1.97 \%$.

240 TEM was performed to identify the formation of core-shell structure, and the size 241 distribution of particles was determined by DLS (Figure IG). The native NaCas appeared as uniform and compact spherical structure (Figure 1A) with an average 243 size of $28 \mathrm{~nm}$. The particle size of Cur@NaCas NPs was slightly larger than that of 244 native NaCas (Figure 1B) with an average size of $32 \mathrm{~nm}$. After the application of a $245 \mathrm{Ca} 3\left(\mathrm{PO}_{4}\right)_{2}$ coating, the formed particles in the range of 195-205 nm, with the TEM 246 image (Figure 1C) revealing a clear core-shell structure. The TEM image shows that 247 the core size of the hybrid NPs ranged from 80 to $120 \mathrm{~nm}$, which is probably due to 248 the aggregation of Cur@NaCas. SEM also demonstrated the dimension change of 249 NPs and the results (Figure 1D-F) consistent with the TEM images. 

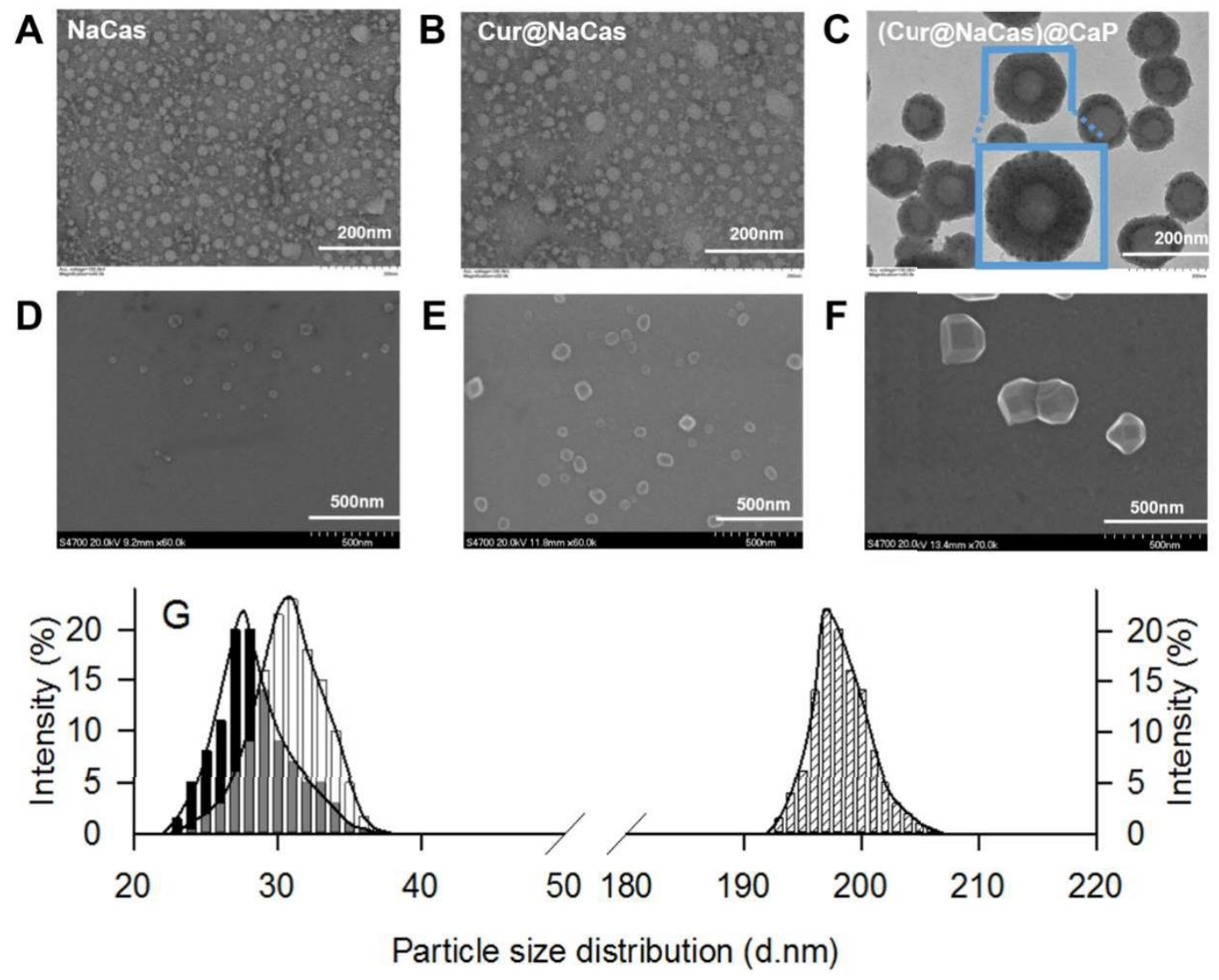

251 Figure 1. Transmission electron microscopy (TEM) (A, B, and C) and scannmg

252 electron microscopy (D, E, and F) and particle size distribution of native NaCas (A,

253 D, G), Cur@NaCas NPs (B, E, G) and (Cur@NaCas)@CaP NPs (C, F, G). G

254 represent the particle size distribution of: native NaCas (black bars ranging from 22 to

$25538 \mathrm{~nm}$ ); Cur@ NaCas (white bars ranging from 22 to $38 \mathrm{~nm}$ ) the grey bars (24 to 36

$256 \mathrm{~nm}$ ) represent the overlapping particle size distribution between native $\mathrm{NaCas}$ and

257 Cur-in-NaCas ; and (Cur@NaCas)@CaP respectively (hatched bars ranging from 192

258 to $208 \mathrm{~nm}$ ). The blue boxed area in (C) shows a high magnification of this region by 259 TEM. 
NaCas@CaP NPs (e) are shown in Figure 2A. The powder diffraction pattern of Cur

264 displayed several sharp peaks at diffraction angles of 8, 8.9, 14.5, 17.2, 21.1, 265 suggesting that Cur existed in a crystalline form.[35, 37] Although NaCas was 266 amorphous lacking crystalline peaks (line ' b'), some minor evidence of crystalline 267 forms persisted in the Cur@NaCas NPs (line ' c' ). The minor crystalline signals might 268 be due to fractions of the entrapped Cur protruding from the $\mathrm{NaCas}$ casing, which is 269 likely to be the result of the spontaneous encapsulation of free Cur by NaCas. 270 Furthermore, the characteristic peak of (Cur@NaCas)@CaP NPs and NaCas@CaP 271 NPs showed the same position with the complete absence of any diffraction peak 272 corresponding to Cur, indicating the formation of (Cur@NaCas)@CaP NPs where the 273 NaCas@CaP core-shell formation completely shielded the encapsulated Cur.

274 FT-IR spectroscopy was performed to verify the interaction between NPs and Cur, 275 and also to confirm the mechanisms of encapsulation and surface coating. The FT-IR 276 spectra of free Cur (a), NaCas (b), Cur@NaCas NPs (c), (Cur@NaCas)@CaP NPs (d) 277 and NaCas@CaP NPs (e) are shown in Figure 2B. Cur was characterized by discrete 278 absorption peaks at $3502 \mathrm{~cm}-\mathrm{I}$ (phenolic $\mathrm{O}-\mathrm{H}$ stretching,) $1720 \mathrm{~cm}-\mathrm{I} \quad(\mathrm{C}=\mathrm{O}$ stretching 279 on the diketone groups), $1614 \mathrm{~cm}^{-1}$ and $1000 \mathrm{~cm}^{-1}(\mathrm{C}=\mathrm{C}$ bending on the aromatic 280 rings $), 1515 \mathrm{~cm}^{-1}\left(\mathrm{C}=\mathrm{C}\right.$ stretching in aromatic ring), $1450 \mathrm{~cm}^{-1}(\mathrm{C}-\mathrm{H}$ bending on 281 methyl groups), and 1300-1200 $\mathrm{cm}^{-1}$ (=C-O-CH 3 stretching of alkyl-aryl ether 282 groups). Most of the characteristic peak of Cur disappeared after encapsulation by 283 NaCas.[38] For NaCas, the characteristic peaks of at $3424 \mathrm{~cm}^{-1}$ (free $\mathrm{O}-\mathrm{H}, \mathrm{N}-\mathrm{H}$ 

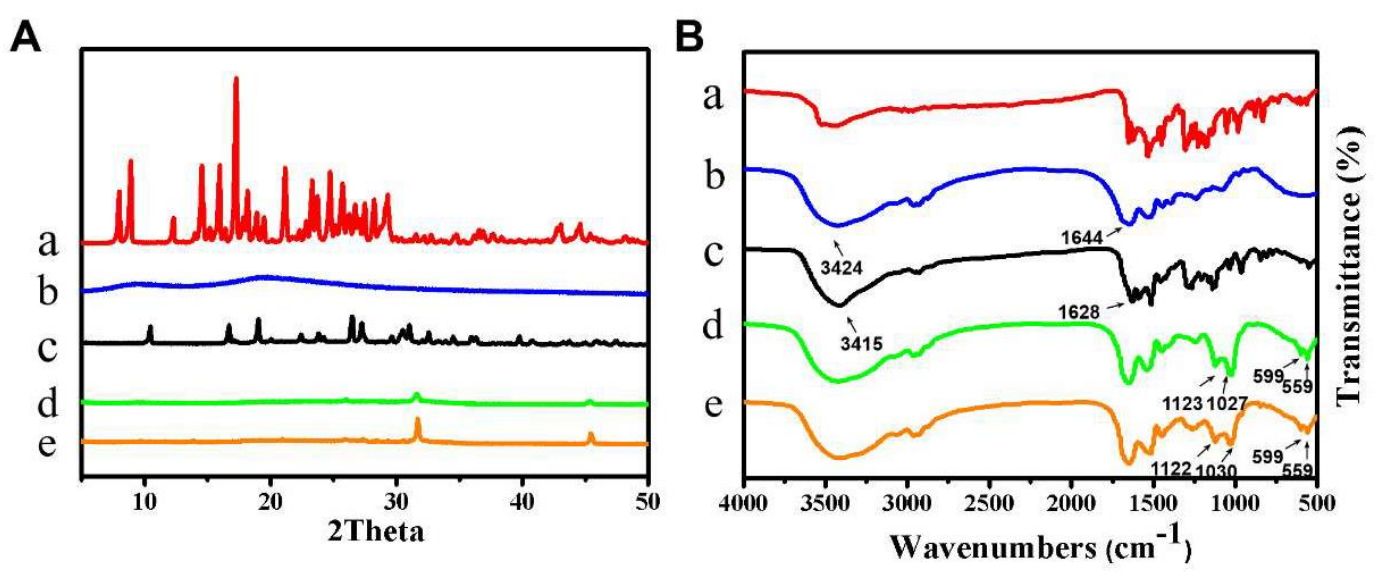

Figure 2. (A) X-ray diffraction patterns and (B) Fourier-transform infrared spectra of the Cur (a), NaCas (b), Cur@NaCas NPs (c), (Cur@NaCas)@CaP NPs (d) and NaCas@CaP NPs (e). core-shell structure (Figure 3A). The zeta-potential values of NaCas decreased from 22.1 to -32.5 after self-assembly with Cur, indicating an enhanced anionic surface 
the number of less negatively charged K-casem and raise the number of high negatively charged casein (as1 -, as2-, -), which increased the overall magnitude of

303 the negative zeta-potential.[41] The zeta potential of $\mathrm{CaP}$ was $-16.4 \mathrm{mV}$, which

304 represents the anionic nature of $\mathrm{CaP}$. Following entrapment of Cur@ CaNas by $305 \mathrm{Ca} 3\left(\mathrm{PO}_{4}\right)_{2}$, the zeta-potential of NPs was marginally increased to $-15.7 \mathrm{mV}$. This 306 confirmed that the shielding by a CaP layer on the surface of Cur@ NaCas altered the 307 negative charge.[42] This higher zeta-potential of (Cur@NaCas)@CaP NPs would be 308 beneficial for its uptake into cells compared with NaCas NPs because of the lower 309 electrostatic repulsion, relative to the negatively charged surfaces of cancer cells, 310 which lowers the barrier to diffuse into cells.[43, 44] This hypothesis has been further 311 investigated in subsequent experiments.

312 Fluorescence spectrum was applied to study the intrinsic fluorescence of Cur as 313 the microenvironment changes in aqueous systems (Figure 3B). At an excitation 314 wavelength of $420 \mathrm{~nm}$, Cur dissolved in ethanol showed the maximum light intensity 315 at $535 \mathrm{~nm}$, while Cur dispersed in water almost without fluorescence. The blue shift 316 of Cur peak at $535 \mathrm{~nm}$, to $517 \mathrm{~nm}$ after encapsulation of NaCas NPs, and the 317 fluorescence intensity increased at the same time. It explained that Cur was loaded 318 into the core of NaCas by hydrophobic interactions, which provided hydrophobic 319 regions for Cur.[45] After the CaP coating, the blue shift of Cur peak from $517 \mathrm{~nm}$ to $320506.2 \mathrm{~nm}$, and the further increased fluorescence intensity indicated that the surface of 321 NaCas was coated by $\mathrm{CaP}$ to form a more hydrophobic matrix. 


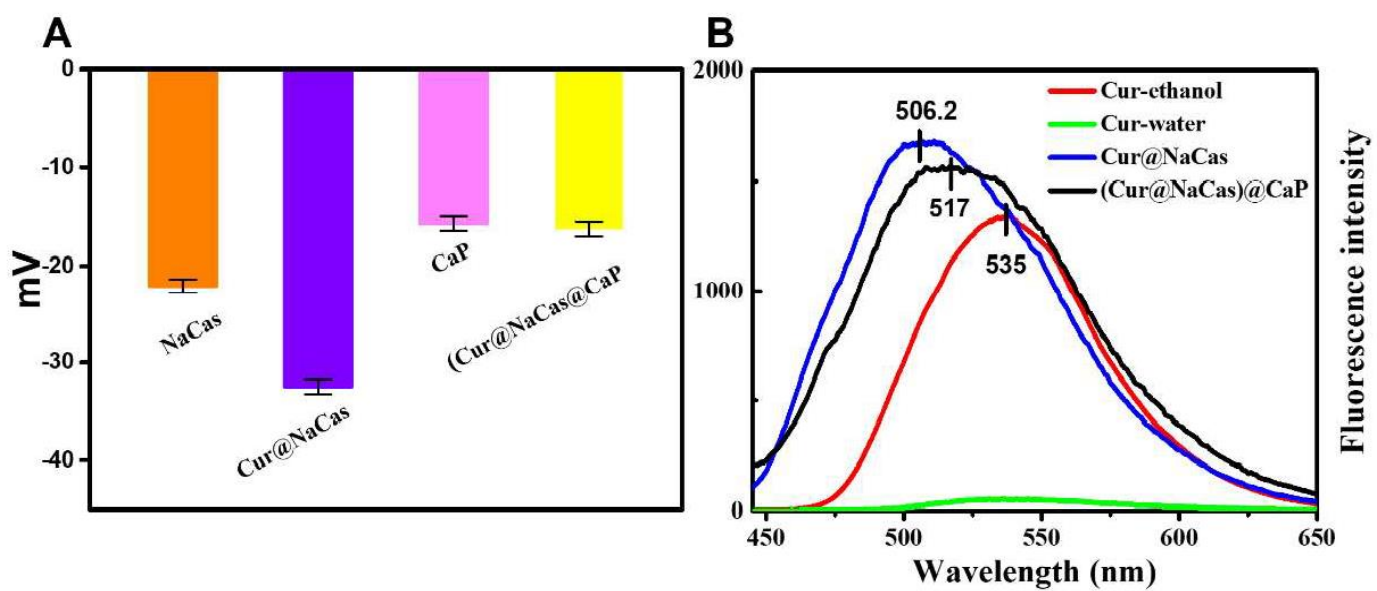

323 Figure 3. (A) Average zeta-potential ofNPs. (B) Fluorescence emission spectra of

324 Cur and NPs.

\subsection{Thermal and UV Stability of (Cur@NaCas)@CaP}

Thermal instability in aqueous solution is a major factor that limits Cur

327 bioavailability.[46] This can be further confirmed in our experiment (Figure 4A). The 328 rest of free Cur was $10.53 \%$ after stored at $80{ }^{\circ} \mathrm{C}$ for $120 \mathrm{~min}$, while $72.9 \%$ of Cur 329 remaining at $25{ }^{\circ} \mathrm{C}$. It was also found that $\mathrm{NaCas}$ encapsulation only limitedly 330 improved the thermal stability of Cur. After encapsulated with $\mathrm{CaP}$ coating, the 331 remaining Cur dramatically reached at $81.54 \%$ under the same heating treatment. 332 Then, we studied the degradation kinetics of free and encapsulated Cur in aqueous 333 solution. Both free and NaCas encapsulated Cur displayed significantly faster 334 degradation at $80^{\circ} \mathrm{C}$ than $25^{\circ} \mathrm{C}$ (Figure 4B and Figure SI ). After heated for 5 min at $33580{ }^{\circ} \mathrm{C}$, more than $60 \%$ of free Cur was lost. However, more than $99 \%$ of Cur loaded 336 in the NaCas@CaP NPs remained. When the heating time reached to $45 \mathrm{~min}, \mathrm{NaCas}$ 337 loaded Cur showed about $50 \%$ of loss. In contrast, about $90 \%$ of the NaCas@ $\mathrm{CaP}$ 
340 photos in Figure 4B). As reported, about $50 \%$ of soy protein isolate loaded Cur was

341 inactivated at $95^{\circ} \mathrm{C}$ within 30 mins,[12] while the Cur encapsulated by NaCas@CaP

342 NPs didn't show sharp reduction within $2 \mathrm{~h}$ under high thermal condition. Thus, the

343 above results indicated that $\mathrm{CaP}$ surface coating could efficiently protect the guest

344 molecules from the thermal degradation.

345 Cur also easily degraded under UV-light.[47] Stored in the UV-light conditions

346 (Figure 4C), free Cur had a sharp degraded by about $81.75 \%$ after storage of $30 \mathrm{~min}$ 347 and more than $90 \%$ of free Cur degraded in 120 min. For NaCas NPs, almost $43 \%$ of 348 loaded Cur degraded after $120 \mathrm{~min}$. With CaP coating on the surface of NaCas, there 349 was a partial degradation by $12 \%$ in $30 \mathrm{~min}$, and then maintained a certainly stability. 350 Obviously, the shell of CaP provided a superior UV protection of at least 1.4 folds 351 than NaCas, and 8 folds better than free Cur.

$352 \quad 3.3$ pH-Responsive Release Comparison of Cur from NaCas with or without CaP $353 \quad$ Coating

354 In practical applications the intend is not to retain the encapsulated compound 355 indefinitely. Instead, the intend would be to facilitate protection of the encapsulated 356 compounds prior to reaching its target, but then have a slow and regular release to 357 limit the rate of physical applications. In this work, the in vitro release experiments of 358 Cur@NaCas NPs and (Cur@NaCas)@CaP NPs in different PBS buffer (pH 7.4 and 
359 5.5) were investigated and shown in Figure 4D. The whole release procedure lasted

$36012 \mathrm{~h}$, and Tween $80(1 \% \mathrm{w} / \mathrm{v})$ was added to enhance the stability of Cur.[48] At both

3617.4 and 5.5, more than $95 \%$ of Cur loaded in NaCas NPs was released within $4 \mathrm{~h}$.

362 Thus, a single protein carrier consisting of NaCas has the capability of facilitating a

$363 \mathrm{pH}-$ response release, however, the relative fast release could cause substantial drug 364 leakage and inactivation. Previous studies have shown that $\mathrm{CaP}$ is also a $\mathrm{pH}$-sensitive

365 material.[31,49] When encapsulated in a NaCas@CaP hybrid matrix, only $30 \%$ of

366 Cur was released at $4 \mathrm{~h}$ in physiological $\mathrm{pH}(\mathrm{pH} 7.4)$, while $66.9 \%$ of Cur was

367 released after $12 \mathrm{~h}$ at $\mathrm{pH}$ 7.4. By contrast, the cumulative release of Cur reached to $36899.8 \%$ after $12 \mathrm{~h}$ at $\mathrm{pH} 5.5$ (Figure 4D). Therefore, the $\mathrm{pH}$-responsive release

369 behavior of NaCas@CaP NPs retards the premature drug release and subsequent rapid

370 degradation/deactivation of the drugs after administration, and achieves controlled

371 release around the acidic microenvironments of tumors sites.[48,50] 
A

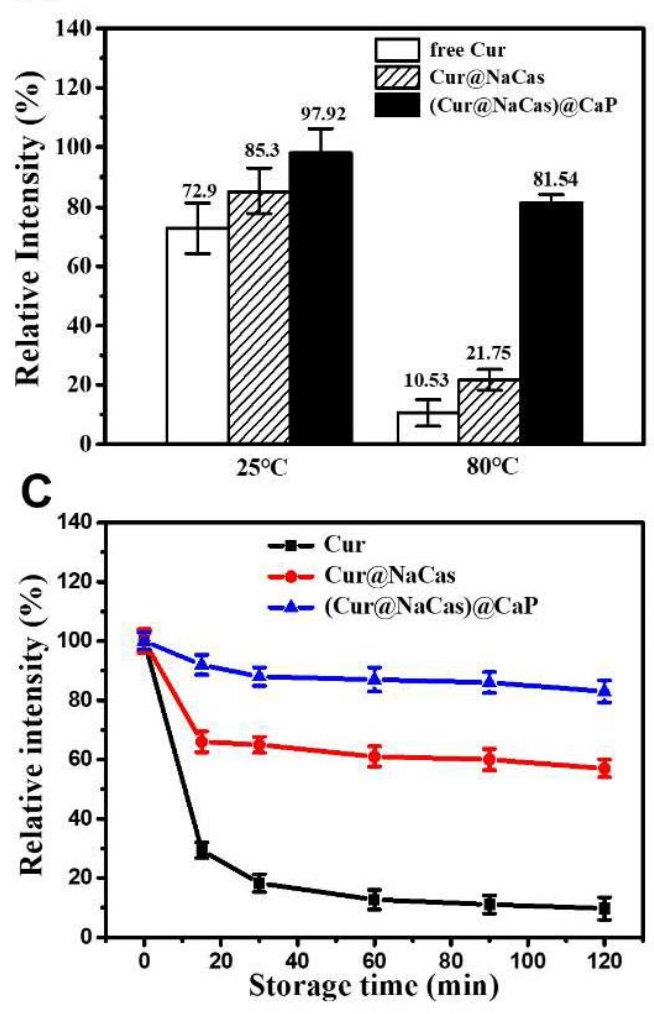

B
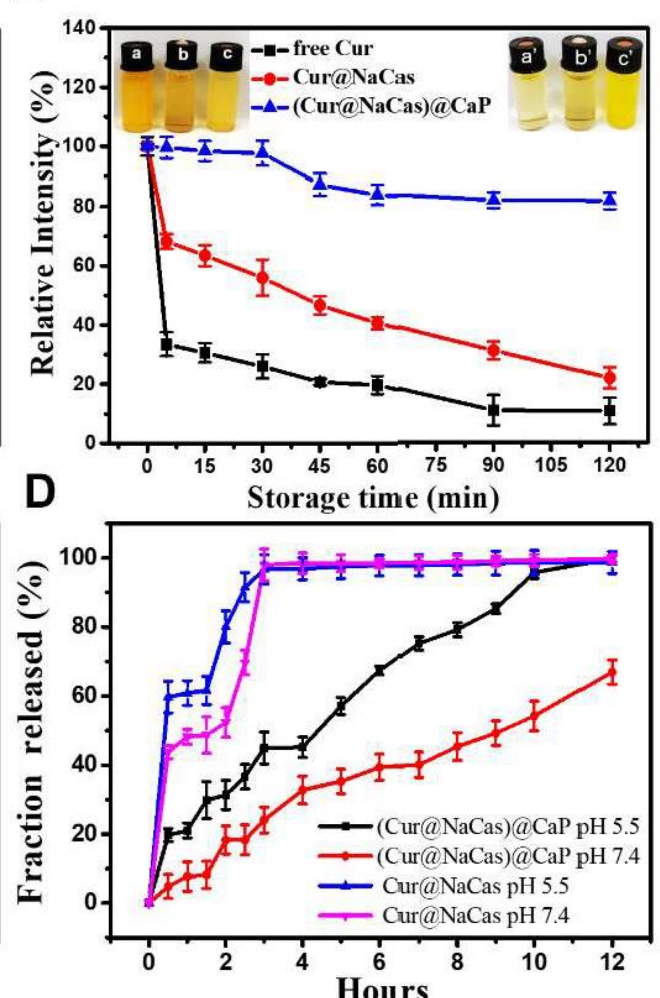
encapsulated compounds have improved cellular uptake capacity. The CLSM images

Figure 4. (A) In vitro stability of free Cur and Cur in the nanoparticles in water at $\mathrm{pH}$ 7.4 upon storage in 120 min at $25{ }^{\circ} \mathrm{C}$ and $80{ }^{\circ} \mathrm{C}$.(B) Degradation kinetics of free Cur and Cur in the NPs in water at $80^{\circ} \mathrm{C}$ from up to $120 \mathrm{~min}$, and the changes of free Cur(a and a') , Cur@NaCas (band b') and (Cur@NaCas)@CaP (c and c' ) before and after heating were shown in the picture.(C) Degradation kinetics of free Cur and Cur in the NPs under ultraviolet light at $25^{\circ} \mathrm{C}$.(D) Release profiles of Cur@NaCas NPs and (Cur@NaCas)@CaP NPs at PBS (pH 7.4 and 5.5) in 12 h.

\subsection{Improved Cellular Uptake ofNaCas@CaP loaded Cur}

Confocal laser scanning microscopy (CLSM) was used to investigate whether through the A549 monolayer cells after incubation with DMSO, free Cur, 
384 Cur@NaCas NPs, and (Cur@NaCas)@CaP NPs (all with equivalent concentration of 385 Cur) are shown in in Figure 5. The commercial fluorescent dye propidium iodide (PI) 386 was used to characterize cell morphology, and the fluorescence of Cur indicates the 387 cell uptake of NPs. (Cur@NaCas)@CaP NPs showed stronger green fluorescent 388 intensity than an equal concentration of free Cur and NaCas loaded Cur, suggesting 389 NaCas@CaP NPs can improve the cellular uptake ability of encapsulated 390 hydrophobic compounds. The reason for the lower green fluorescent intensity for the 391 Cur@NaCas NPs might be due to leakage of Cur from Cur@NaCas NPs. Free Cur 392 was shown to degrade rapidly and as such reduce its bioavailability at a slightly acidic 393 cancer cell environment pH (Figure S2). For the (Cur@NaCas)@CaP NPs, the 394 presence of a $\mathrm{CaP}$ shell increased the stability of Cur in a physiological aqueous 395 environment and reduced the cell uptake barriers between NPs and Cur (Figure 5), 396 thus evidencing improved cellular uptake and stability.[51, 52] On the other hand,Cur 397 would be released from the NaCas core of the (Cur@NaCas)@CaP NPs following 398 cellular uptake. It is likely that any intracellular release would have been in part 399 facilitated by endosomes and lysosomes, and achieved a cumulative intracellular 400 release.[53] Thus, the synergistic effect of shell and core increased the cell uptake 401 capacity of NPs and achieved efficient intracellular release. 

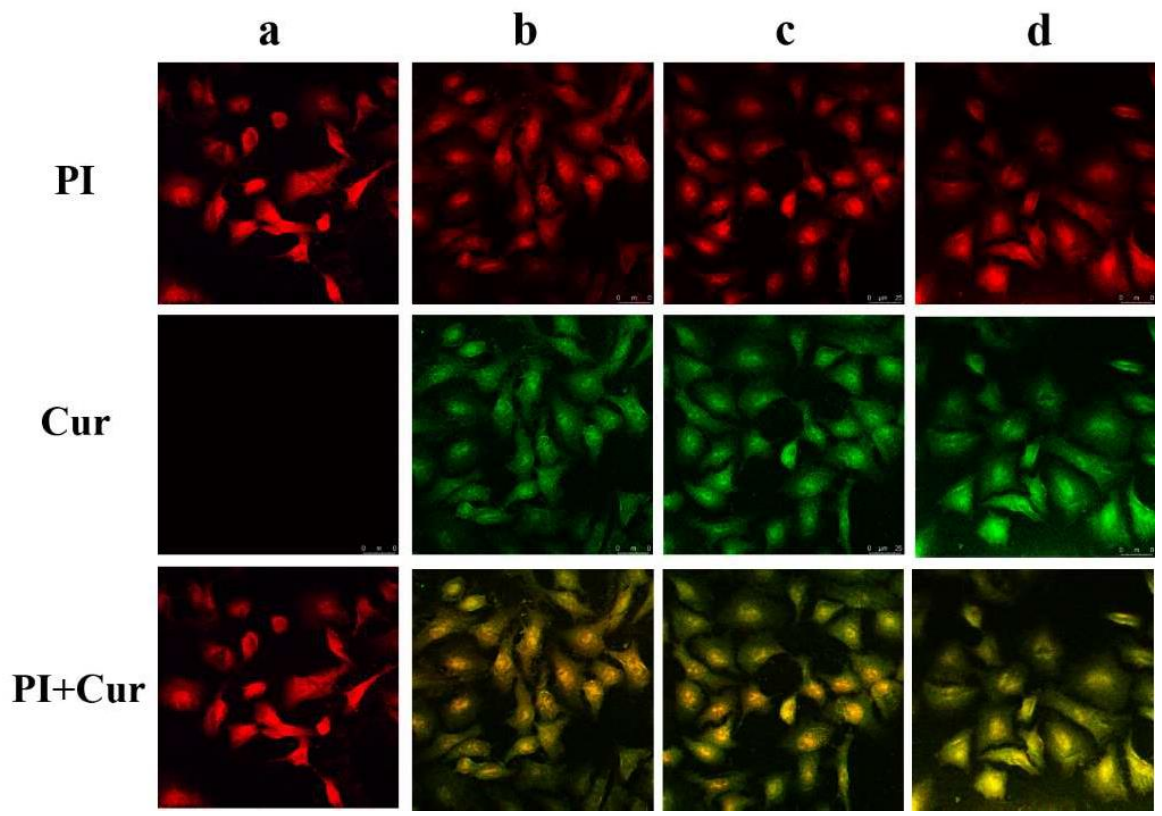

403 Figure 5. Uptake of free Cur and encapsulated Cur into epithelial cells (A549, human

404 alveolar basal epithelial cells) as observed by confocal laser scanning microscopy

405 (CLSM) afte 4h of exposure. Column ' PI', Propidium iodide (PI)-only control;

406 Column ' Cur', Cur-only control; Column ' PI + Cur', cells treated with both PI and

407 Cur. (a) cells treated with DMSO; (b) cells treated with free Cur; (c) cells treated with

408 Cur@NaCas NPs; and (d) cells treated with (Cur@NaCas)@CaP NPs

$409 \quad 3.5$ Cellular Antioxidant Activity

410 It has previously been shown that Cur has antioxidant activity and free radical

411 scavenging activities.[47] The cellular anti-oxidant activity (CAA) of free Cur and

412 encapsulated Cur were assessed by the MGC-803 cell model (Figure 6A), and the

413 anti-proliferation activities of NPs on MGC-803 cells are shown in Figure S3.

414 According to the principle of CAA, peroxy radicals produced by ABAP cause

415 oxidation of DCFH to form the fluorescent DCF, the DCF fluorescence intensity

416 reflects the degree of oxidation damage caused by free radicals.[35] The antioxidant 
417 capacity of any natural compounds can be reflected by the reduced DCF fluorescence

418 intensity. The results (Figure 6A) indicated that DCF accumulated continuously in

419 the cells over time, while Cur could quench free radicals to reduce the concentration

420 of intracellular DCF. The reduced accumulation of DCF demonstrates that both the

421 added Cur@NaCas NPs and (Cur@NaCas)@CaP NPs have antioxidant activity,

422 which is consistent with previous work that reported that Cur loaded inclusion

423 complexes possessed a higher free radical scavenging capacity compared with free

424 Cur.[54] The CAA value were determined using eq.(3). The CAA results indicate that 425 the NaCas and NaCas@CaP NPs loaded Cur had better cellular anti-oxidant activity 426 than free Cur (Figure 6B).

A

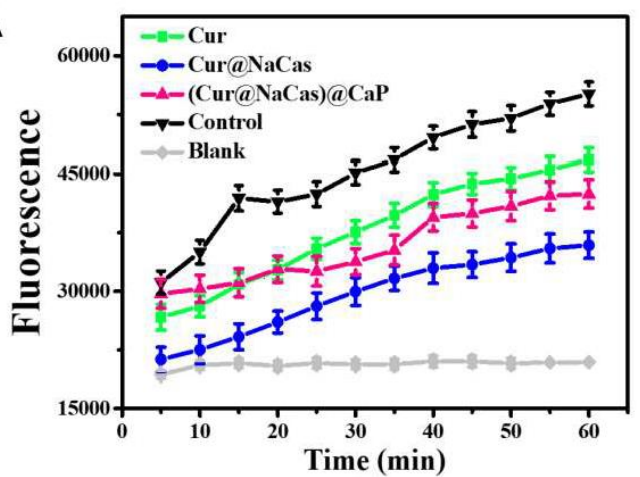

C

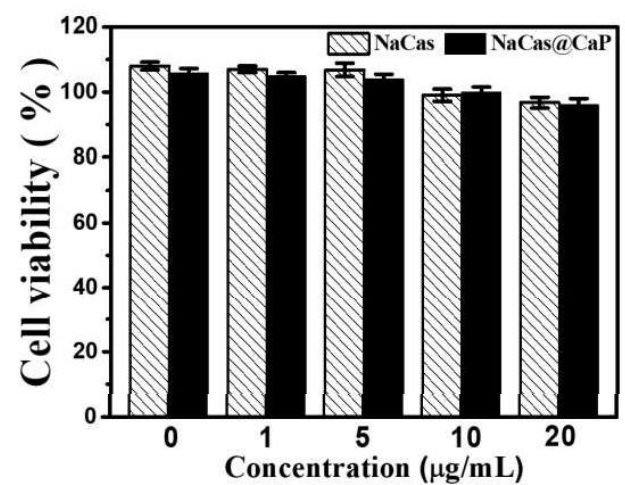

B
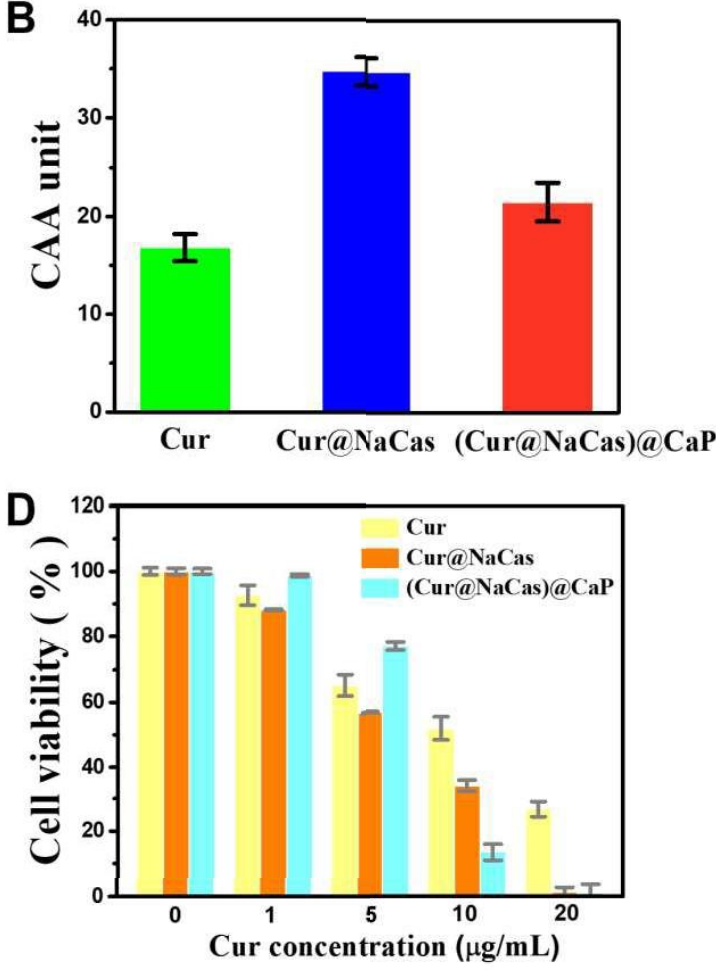

Figure 6. (A) The kinetics curve of DCF fluorescence from cellular anti-oxidant activity (CAA) of Cur, Cur@NaCas, (Cur@NaCas)@CaP, control, and blank sample. 
In all instances the cells were exposed to equal amount of Cur. Error bars indicate the standard deviation following triplicate determinations. (B)The comparison of cellular anti-oxidant activity value for different samples. (C) Anti-proliferation activity the blank NPs toxicity of human alveolar basal epithelial cells (A549 cells). (D) Antiproliferation activity of 1, 5, 10 and $20 \mu \mathrm{g} / \mathrm{mL}$ DMSO-dissolved and encapsulated Cur against A549 cells.

\subsection{Cell Viability}

In order to investigate the cytotoxicity of (Cur@NaCas)@CaP NPs in cancer cells, we monitored the cell proliferation using human alveolar basal epithelial cancer cells (A549 cells) as a model. The native NaCas and NaCas@CaP NPs (without Cur) showed almost $100 \%$ cell viability at $72 \mathrm{~h}$ (Figure 6C), indicating that the blank nanoparticle was nontoxic and biocompatible with the cell model used in these experiments.[55] However, with Cur encapsulated in either NaCas or NaCas@CaP NPs showed an improved anti-proliferation activity against A549 cancer cells when compared with free Cur (Figure 6D). When the concentration of free Cur was applied at $1 \mu \mathrm{g} / \mathrm{mL}$ and $5 \mu \mathrm{g} / \mathrm{mL}$, it showed a mean cell viability of $92.6 \%$ and $65.2 \%$ respectively; while the Cur@NaCas inclusion complex showed a mean cell viability of $88.2 \%$ and $56.6 \%$ at $1 \mu \mathrm{g} / \mathrm{mL}$ and $5 \mu \mathrm{g} / \mathrm{mL}$ of free Cur equivalent respectively. The (Cur@NaCas)@CaP inclusion complex did not show the same enhanced antiproliferation effect on A549 cells compared to free Cur at the same Cur concentration, with a mean cell viability of $98.8 \%$ and $77.0 \%$ at $1 \mu \mathrm{g} / \mathrm{mL}$ and $5 \mu \mathrm{g} / \mathrm{mL}$ of free Cur 
equivalent respectively. A more prominent improvement in the anti-proliferation activity of NaCas@CaP loaded Cur was observed at an effective dose of free Cur 10 $\mu \mathrm{g} / \mathrm{mL}$, with a mean cell viability of $13.5 \%$. At the same effective Cur concentration, 454 the cell viability of free Cur and Cur@NaCas NPs was $51.7 \%$ and $34.0 \%$. It appears that the anticancer effect of NPs loaded with Cur was related to the release property and biocompatibility of NPs, which is also relevant to the instability, insolubility and 457 concentration of Cur.[42, 56] More specifically, when considering Cur at a lower 458 concentration, the release rate of Cur from the NPs was less than the rate of 459 deg radation, which resulted in a lower anti-cancer activity of the encapsulated Cur 460 compared to free Cur. At the higher concentration, free Cur gradually degraded in 461 aqueous conditions in which the experiments were carried out, due to Cur' sinstability 462 and low biocompatibility; This while the chemical stability of NPs loaded Cur at the 463 same equivalent concentrations was remarkably enhanced (Figure 6D). It appears that 464 the NaCas@CaP loaded Cur achieved a more continuous anti-cancer effect, which 465 agrees with the CLSM results in this research (Figure 5). At the highest concentration 466 tested $(20 \mu \mathrm{g} / \mathrm{mL})$ in this study, the cell viability of A549 cells exposed to both 467 Cur@NaCas loaded and (Cur@NaCas)@CaP loaded NPs was almost non-existent, 468 while the addition of an equivalent concertation of free Cur saw a viability of 469 approximately $30 \%$. This could be ascribed to the degradation of free Cur under the 470 experimental conditions where some of the added free Cur might have already 471 degraded before its full weight of the higher concentration could affect the cells 
472 (Figure S2), plus, it might have been possible that the higher concentration saturated

473 the anti-proliferation effect against A549 cancer cells.

\section{4. Conclusions}

475 In conclusion, a novel hybrid nanodelivery system was prepared using NaCas and

$476 \mathrm{CaP}$. The nanosized NaCas@CaP NPs were shown to have an obvious core-shell

477 structure, which markedly improved the stability of Cur under thermal conditions and

478 UV light conditions. Besides, the $\mathrm{pH}$-responsive release of Cur around cancercell

479 environment was achieved. Furthermore, the results proved that NaCas@CaP NPs can

480 improve anti-oxidant activity, cell uptake ability and anti-proliferative activity of Cur

481 in living cells. Our research provided a simple and stable nano-platform for

482 encapsulating sensitive hydrophobic biomolecules. The results revealed that this core-

483 shell nanodelivery system was meaningful for keeping bioactivities of sensitive

484 molecules and improving the bioavailability in cancer treatment. Thus, this

485 nanodelivery system is expected to be applied for in food and pharmaceutical

486 products field.

487 For human health and practical application, further research is still undergoing in

488 our laboratory including the delivery behavior and uptake mechanism of this

489 nanodelivery system in various food and animals' systems, as well as the bioactivities

490 of encapsulated molecules after the delivery of gastrointestinal tract. 
The first two authors have equal contributions

495

496

497

498

499

500

501

502

503

504

505

506

507

508

509

510

511

512

513

514

515

516

517

518

519

520

521

522

523

524

525

Notes

The authors declare no competing financial interest.

\section{ACKNOWLEDGMENT}

The authors acknowledged financial support from the National Natural Science

Foundation of China (21878014), the Double First-rate Program (ylkxj03), and the

111 Project (B13005).

\section{REFERENCES}

[1] X. Duan, H. Chen, L. Fan, J. Kong, Drug Self-Assembled Delivery System with Dual Responsiveness for Cancer Chemotherapy, ACS Biomater Sci Eng 2(12) (2016) 2347-2354.

[2] Y. Li, Y. Wang, G. Huang, J. Gao, Cooperativity Principles in Self-Assembled Nanomedicine, Chem Rev 118(11) (2018) 5359-5391.

[3] W. Cui, J. Li, G. Decher, Self-Assembled Smart Nanocarriers for Targeted Drug Delivery, Adv Mater 28(6) (2016) 1302-11.

[4] M.H. Li, Z. Luo, Y.L. Zhao, Self-Assembled Hybrid Nanostructures: Versatile Multifunctional Nanoplatforms for Cancer Diagnosis and Therapy, Chem Mater 30(1) (2018) 25-53.

[5] L. Zhou, T. Qiu, F. Lv, L. Liu, J. Ying, S. Wang, Self-Assembled Nanomedicines for Anticancer and Antibacterial Applications, Adv Healthc Mater 7(20) (2018) e1800670.

[6] Y.M.a.H. Maeda, A New Concept for Macromolecular Therapeutics in Cancer Chemotherapy: Mechanism of Tumoritropic Accumulation of Proteins and the Antitumor Agent Smancsl, Cancer Res 46 (1986) 6387-6392.

[7] Y. Jin, R. Xin, P. Ai, D. Chen, Self-assembled drug delivery systems 2. Cholesteryl derivatives of antiviral nucleoside analogues: synthesis, properties and the vesicle formation, Int J Pharm 350(1-2) (2008) 330-7.

[8] D. Kalyane, N. Raval, R. Maheshwari, V. Tambe, K. Kalia, R.K. Tekade, Employment of enhanced permeability and retention effect (EPR): Nanoparticlebased precision tools for targeting of therapeutic and diagnostic agent incancer, Mater Sci Eng C 98 (2019) 1252-1276. 
[9] S.Y. Qin, A.Q. Zhang, S.X. Cheng, L. Rong, X.Z. Zhang, Drug self-delivery systems for cancer therapy, Biomaterials 112 (2017) 234-247.

[1O] S. Jaiswal, P. Mishra, Co-delivery of curcumin and serratiopeptidase in HeLa and MCF-7 cells through nanoparticles show improved anti-cancer activity, Mater Sci Eng C 92 (2018) 673-684.

[11] H. Lu, Q. Wang, G. Li, Y. Qiu, Q. Wei, Electrospun water-stable zein/ethyl cellulose composite nanofiber and its drug release properties, Mater Sci Eng C 74 (2017) 86-93.

[12] F.P. Chen, B.S. Li, C.H. Tang, Nanocomplexation between curcumin and soy protein isolate: influence on curcumin stability/bioaccessibility and in vitro protein digestibility, J Agric Food Chem 63(13) (2015) 3559-69.

[13] W. Kajthunyakarn, D. Sakloetsakun, T. Pongjanyakul, Sodium caseinatemagnesium aluminum silicate nanocomposite films for modified-release tablets, Mater Sci Eng C 92 (2018) 827-839.

[14] R. Li, Y. Ma, Y. Dong, Z. Zhao, C. You, S. Huang, X. Li, F. Wang, Y. Zhang, Novel Paclitaxel-Loaded Nanoparticles Based on Human H Chain Ferritin for TumorTargeted Delivery, ACS Biomater Sci Eng 5 (2019) 6645-6654.

[15] K. Pan, H. Chen, S.J. Baek, Q. Zhong, Self-assembled curcumin-soluble soybean polysaccharide nanoparticles: Physicochemical properties and in vitro antiproliferation activity against cancer cells, Food Chem 246 (2018) 82-89.

[16] Y. Zhang, Q.X. Zhong, Encapsulation of bixin in sodium caseinate to deliver the colorant in transparent dispersions, Food Hydrocoll 33(1) (2013) 1-9.

[17] 0. Menendez-Aguirre, A. Kessler, W. Stuetz, T. Grune, J. Weiss, J. Hinrichs, Increased loading of vitamin D2 in reassembled casein micelles with temperaturemodulated high pressure treatment, Food Res Int 64 (2014) 74-80.

[18] R. Penalva, I. Esparza, M. Agueros, C.J. Gonzalez-Navarro, C. GonzalezFerrero, J.M. Irache, Casein nanoparticles as carriers for the oral delivery of folic acid, Food Hydrocoll 44 (2015) 399-406.

[19] A. Rashidinejad, S.M. Loveday, G.B. Jameson, J.P. Hindmarsh, H. Singh, Rutincasein co-precipitates as potential delivery vehicles for flavonoid rutin, Food Hydrocoll 96 (2019) 451-462.

[20] A. Bahri, M. Martin, C. Gergely, S. Marchesseau, D. Chevalier-Lucia, Topographical and nanomechanical characterization of casein nanogel particles using atomic force microscopy, Food Hydrocoll 83 (2018) 53-60.

[21] C. Moitzi, I. Portnaya, 0. Glatter, 0. Ramon, D. Danino, Effect of Temperature on Self-Assembly of Bovine -Casein above and below Isoelectric pH. Structural Analysis by Cryogenic-Transmission Electron Microscopy and Small-Angle X-ray Scattering, Langmuir 24(7) (2008) 3020-3029.

[22] M. Li, M.A.E. Auty, S.V. Crowley, A.L. Kelly, J.A. O'Mahony, A. Brodkorb, Self-association of bovine -casein as influenced by calcium chloride, buffer type and temperature, Food Hydrocoll 88 (2019) 190-198.

[23] Y. Luo, K. Pan, Q. Zhong, Casein/pectin nanocomplexes as potential oral delivery vehicles, Int J Pharm 486(1-2) (2015) 59-68.

[24] Q. Hu, M. Bae, E. Fleming, J.-Y. Lee, Y. Luo, Biocompatible polymeric 
570 nanoparticles with exceptional gastrointestinal stability as oral delivery vehicles for

571 lipophilic bioactives, Food Hydrocoll, 89 (2019) 386-395..

572 [25] C. Sun, Y. Gao, Q. Zhong, Properties of Ternary Biopolymer Nanocomplexes of 573 Zein, Sodium Caseinate, and Propylene Glycol Alginate and Their Functions of

$574 \quad$ Stabilizing High Internal Phase Pickering Emulsions, Langmuir 34(31) (2018) 9215-

5759227.

576 [26] H. Zhang, Y. Fu, Y. Xu, F. Niu, Z. Li, C. Ba, B. Jin, G. Chen, X. Li, One-step

577 assembly of zein/caseinate/alginate nanoparticles for encapsulation and improved

578 bioaccessibility of propolis, Food Funct 10(2) (2019) 635-645.

579 [27] M. Yerramilli, S. Ghosh, Long-term stability of sodium caseinate-stabilized

580 nanoemulsions, J Food Sci Technol 54(1) (2017) 82-92.

581 [28] Q. Hu, M. Bae, E. Fleming, J.-Y. Lee, Y. Luo, Biocompatible polymeric

582 nanoparticles with exceptional gastrointestinal stability as oral delivery vehicles for 583 lipophilic bioactives, Food Hydrocoll 89 (2019) 386-395.

584 [29] H.B. Yao, M.R. Gao, S.H. Yu, Small organic molecule templating synthesis of 585 organic-inorganic hybrid materials: their nanostructures and properties, Nanoscale $586 \quad 2(3)(2010)$ 323-34.

587 [30] E. Soo, S. Thakur, Z. Qu, S. Jambhrunkar, H.S. Parekh, A. Popat, Enhancing

588 delivery and cytotoxicity of resveratrol through a dual nanoencapsulation approach, $\mathbf{J}$ 589 Colloid Interface Sci 462 (2016) 368-74.

590 [31] P. Mi, D. Kokuryo, H. Cabral, H. Wu, Y. Terada, T. Saga, I. Aoki, N. 591 Nishiyama, K. Kataoka, A pH-activatable nanoparticle with signal-amplification 592 capabilities for non-invasive imaging of tumour malignancy, Nat Nanotechnol 11(8) 593 (2016) 724-30.

594 [32] F. Li, Q. Xing, Y. Han, Y. Li, W. Wang, T.S.H. Perera, H. Dai, Ultrasonically 595 assisted preparation of poly(acrylic acid)/calcium phosphate hybrid nanogels as $\mathrm{pH}$ 596 responsive drug carriers, Mater Sci Eng C 80 (2017) 688-697.

597 [33] Q. Song, L. Li, K. Xiong, W. Tian, J. Lu, J. Wang, N. Huang, Q. Tu, Z. Yang, A 598 facile dopamine-mediated metal-catecholamine coating for therapeutic nitric oxide 599 gas interface-catalytic engineering of vascular devices, Biomater Sci 7(9) (2019) $6003741-3750$.

601 [34] M. Canillas, P. Pena, A.H. de Aza, M.A. Rodriguez, Calcium phosphates for 602 biomedical applications, Boletin de la Sociedad Espanola de Ceramica y Vidrio 56(3) 603 (2017) 91-112.

604 [35] Z. Liu, Q. Wu, J. He, F. Vriesekoop, H. Liang, Crystal-Seeded Growth of pH605 Responsive Metal-Organic Frameworks for Enhancing Encapsulation, Stability, and 606 Bioactivity of Hydrophobicity Compounds, ACS Biomater Sci Eng 5 (2019) 65816076589.

608 [36] A.F. Martins, P.V. Bueno, E.A. Almeida, F.H. Rodrigues, A.F. Rubira, E.C. 609 Muniz, Characterization of N-trimethyl chitosan/alginate complexes and curcurnin 610 release, Int $\mathbf{J}$ Biol Macromol 57 (2013) 174-84.

611 [37] J. Wu, J. Wang, J. Zhang, Z. Zheng, D.L. Kaplan, G. Li, X. Wang, Oral Delivery 612 of Curcumin Using Silk Nano- and Microparticles, ACS Biomater Sci Eng 4(11) 613 (2018) 3885-3894. 
614 [38] H. Xiang, D. Sun-Waterhouse, C. Cui, W. Wang, K. Dong, Modification of soy 615 protein isolate by glutaminase for nanocomplexation with curcumin, Food Chem 268 616 (2018) 504-512.

617 [39] H.Q. Chen, Q.X. Zhong, Processes improving the dispersibility of spray-dried

618 zein nanoparticles using sodium caseinate, Food Hydrocoll 35 (2014) 358-366.

619 [40] W.-H. Lee, C.-Y. Loo, R. Rohanizadeh, Functionalizing the surface of 620 hydroxyapatite drug carrier with carboxylic acid groups to modulate the loading and 621 release of curcumin nanoparticles, Mater Sci Eng C 99 (2019) 929-939.

622 [41] K. Pan, H. Chen, P.M. Davidson, Q. Zhong, Thymol nanoencapsulated by 623 sodium caseinate: physical and antilisterial properties, J Agric Food Chem 62(7) 624 (2014) 1649-57.

625 [42] B. Deng, M. Xia, J. Qian, R. Li, L. Li, J. Shen, G. Li, Y. Xie, Calcium 626 Phosphate-Reinforced Reduction-Sensitive Hyaluronic Acid Micelles for Delivering 627 Paclitaxel in Cancer Therapy, Mol Pharm 14(6) (2017) 1938-1949.

628 [43] A. Verma, F. Stellacci, Effect of Surface Properties on Nanoparticle-Cell

629 Interactions, Small 6(1) (2010) 12-21.

630 [44] Z. Zhou, Y. Shen, J. Tang, E. Jin, X. Ma, Q. Sun, B. Zhang, E.A. Van Kirk, W.J. 631 Murdoch, Linear polyethyleneimine-based charge-reversal nanoparticles for nuclear632 targeted drug delivery, J Mater Chem 21(47) (2011) 19114-19123.

633 [45] K. Pan, Q. Zhong, S.J. Baek, Enhanced dispersibility and bioactivity of curcumin 634 by encapsulation in casein nanocapsules, J Agric Food Chem 61(25) (2013) 6036-43.

635 [46] M. Kharat, Z. Du, G. Zhang, D.J. McClements, Physical and Chemical Stability

636 of Curcumin in Aqueous Solutions and Emulsions: Impact of pH, Temperature, and

637 Molecular Environment, J Agric Food Chem 65(8) (2017) 1525-1532.

638 [47] Z. Hussain, H.E. Thu, M.W. Amjad, F. Hussain, T.A. Ahmed, S. Khan, 639 Exploring recent developments to improve antioxidant, anti-inflammatory and 640 antimicrobial efficacy of curcumin: A review of new trends and future perspectives, 641 Mater Sci Eng C 77 (2017) 1316-1326.

642 [48] M. Zheng, S. Liu, X. Guan, Z. Xie, One-Step Synthesis of Nanoscale Zeolitic 643 Imidazolate Frameworks with High Curcumin Loading for Treatment of Cervical 644 Cancer, ACS Appl Mater Interfaces 7(40) (2015) 22181-7.

645 [49] P. Mi, N. Dewi, H. Yanagie, D. Kokuryo, M. Suzuki, Y. Sakurai, Y. Li, I. Aoki, 646 K. Ono, H. Takahashi, H. Cabral, N. Nishiyama, K. Kataoka, Hybrid Calcium 647 Phosphate-Polymeric Micelles Incorporating Gadolinium Chelates for Imaging648 Guided Gadolinium Neutron Capture Tumor Therapy, ACS Nano 9(6) (2015) 5913-

6495921.

650 [50] L. Liu, J. Guo, P. Liu, Raspberry-Shaped Independent Temperature and pH Dual-

651 Responsive CPMAA@CPNIPAM Yolk/Shell Microspheres for Site-Specific

652 Targeted Delivery of Anticancer Drugs, Ind Eng Chem Res 55(16) (2016) 4790-4796.

653 [51] Y. Luo, Z. Teng, T.T. Wang, Q. Wang, Cellular uptake and transport of zein

654 nanoparticles: effects of sodium caseinate, J Agric Food Chem 61(31) (2013) 7621-9.

655 [52] P. Das, N.R. Jana, Length-Controlled Synthesis of Calcium Phosphate Nanorod

656 and Nanowire and Application in Intracellular Protein Delivery, ACS Appl Mater

657 Interfaces 8(13) (2016) 8710-20. 
658 [53] S.J. Kaczmarczyk, K. Sitaraman, H.A. Young, S.H. Hughes, D.K. Chatterjee, 659 Protein delivery using engineered virus-like particles, Proc Natl Acad Sci U S A $660108(41)$ (2011) 16998-7003.

661 [54] P. Jiang, J. Huang, C. Bao, L. Jiao, H. Zhao, Y. Du, F. Ren, Y. Li, Enzymatically 662 partial hydrolyzed a-lactalbumin peptides for self-assembled micelles formation and 663 their application for coencapsulation of multiple anti-oxidants, J Agric Food Chem 66 664 (2018) 12921-12930.

665 [55] W.H. Lee, C.Y. Loo, R. Rohanizadeh, Functionalizing the surface of 666 hydroxyapatite drug carrier with carboxylic acid groups to modulate the loading and 667 release of curcumin nanoparticles, Mater Sci Eng C 99 (2019) 929-939.

668 [56] B. Lei, M. Wang, Z. Jiang, W. Qi, R. Su, Z. He, Constructing Redox-Responsive 669 Metal-Organic Framework Nanocarriers for Anticancer Drug Delivery, ACS Appl 670 Mater Interfaces 10(19) (2018) 16698-16706. 


\section{Declaration of interests}

D The authors declare that they have no known competing financial interests or personal relationships that could have appeared to influence the work reported in this paper.

$\square$ The authors declare the following financial interests/personal relationships which may be considered as potential competing interests: 


\section{Author Statement}

Hao Liang:

Conceptualization, methodology, resources, project administration,lwriting - original draft, writing - review \& editing, supervision, funding acquisition

Qiao Wu:

Conceptualization, methodology, validation, formal analysis, investigation, data curation, writing - original draft

Huiling Gao

Conceptualization, methodology, supervision

Frank Vriesekoop

Formal analysis, writing - original draft, writing - review \& editing

Zexun Liu

Methodology, resources.

Jie $\mathrm{He}$

Methodology 


\section{Supporting Information}

\section{Calcium Phosphate Coated Core-Shell Protein Nanocarriers: Robust \\ Stability, Controlled Release and Enhanced Anticancer Activity for \\ Curcumin Delivery}

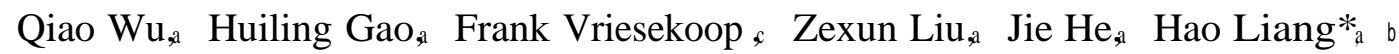

a State Key Laboratory of Chemical Resource Engineering, Beijing University of

Chemical Technology, Beijing100029, P.R. China

b Qinhuangdao Bohai Biological Research Institute of Beijing University of Chemical

Technology, Qinhuangdao 066000, China

c Department of Food Technology and Innovation, Harper Adams University,

Newport TFl0 8NB, Shropshire, United Kingdom.

Corresponding Author

* E-mail: lianghao@rnail.buct.edu.cn 


\section{Supplementary tables}

Table S1 Effect of NaCas concentration on drug loading efficiency (DLE) (\%).

\begin{tabular}{ccccc}
\hline NaCas $(\mathrm{mg} / \mathrm{mL})$ & Cur $(\mathrm{mg} / \mathrm{mL})$ & Stirring speed $(\mathrm{rpm} / \mathrm{min})$ & Stirring time $(\mathrm{h})$ & DLE $(\%)$ \\
\hline 0.5 & 0.5 & 600 & 1.5 & $62.34 \pm 1.30$ \\
0.75 & 0.5 & 600 & 1.5 & $78.67 \pm 1.14$ \\
$\mathbf{1}$ & 0.5 & 600 & 1.5 & $80.63 \pm 1.37$ \\
1.25 & 0.5 & 600 & 1.5 & $80.94 \pm 1.43$ \\
1.5 & 0.5 & 600 & 1.5 & $81.13 \pm 1.29$ \\
\hline
\end{tabular}

All samples were prepared at room temperature.

Table S2 Effect of stirring speed on DLE (\%)

\begin{tabular}{ccccc}
\hline NaCas $(\mathrm{mg} / \mathrm{mL})$ & Cur $(\mathrm{mg} / \mathrm{mL})$ & Stirring speed $(\mathrm{rpm} / \mathrm{min})$ & Stirring time $(\mathrm{h})$ & DLE $(\%)$ \\
\hline $\mathbf{1}$ & 0.5 & 400 & 1.5 & $57.83 \pm 1.17$ \\
$\mathbf{1}$ & 0.5 & 500 & 1.5 & $63.49 \pm 1.23$ \\
$\mathbf{1}$ & 0.5 & 600 & 1.5 & $80.98 \pm 1.27$ \\
$\mathbf{1}$ & 0.5 & 700 & 1.5 & $84.24 \pm 1.31$ \\
$\mathbf{1}$ & 0.5 & 800 & 1.5 & $84.32 \pm 1.43$ \\
\hline
\end{tabular}

All samples were prepared at room temperature.

Table S3 Effect of stirring time on DLE (\%)

\begin{tabular}{ccccc}
\hline NaCas $(\mathrm{mg} / \mathrm{mL})$ & Cur $(\mathrm{mg} / \mathrm{mL})$ & Stirring speed $(\mathrm{rpm} / \mathrm{min})$ & Stirring time $(\mathrm{h})$ & DLE $(\%)$ \\
\hline $\mathbf{1}$ & 0.5 & 700 & 0.5 & $50.64 \pm 1.34$ \\
$\mathbf{1}$ & 0.5 & 700 & 1.0 & $73.68 \pm 1.49$ \\
$\mathbf{1}$ & 0.5 & 700 & 1.5 & $84.97 \pm 1.38$ \\
$\mathbf{1}$ & 0.5 & 700 & 2.0 & $90.01 \pm 1.29$ \\
$\mathbf{1}$ & 0.5 & 700 & 2.5 & $90.27 \pm 1.44$ \\
\hline
\end{tabular}

All samples were prepared at room temperature. 
Table S4 Effect of the added CaP volume on DLE (\%)

\begin{tabular}{cccc}
\hline CaP concentration $(\mathrm{mM})$ & $\mathrm{CaP}$ volume $(\mu \mathrm{L})$ & Mixing time $(\mathrm{min})$ & DLE $(\%)$ \\
\hline 50 & 5 & 2 & $21.57 \pm 2.06$ \\
50 & 10 & 2 & $53.24 \pm 1.92$ \\
50 & 15 & 2 & $80.42 \pm 2.14$ \\
50 & 20 & 2 & $99.25 \pm 1.97$ \\
50 & 25 & 2 & $99.47 \pm 2.03$ \\
\hline
\end{tabular}

All samples were prepared at room temperature. 
Supplementary figures

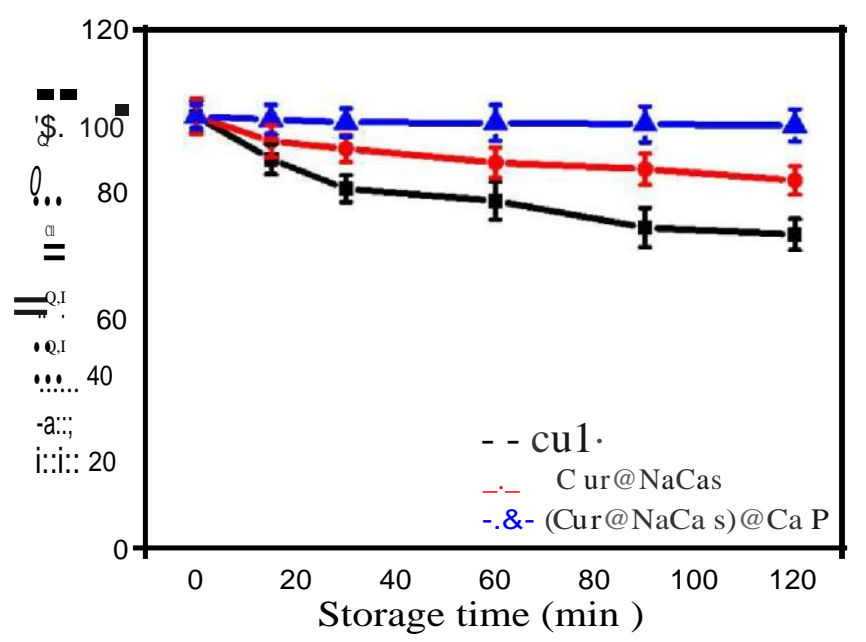

Figure S1 Degradation kinetics of free Cur and Cur in the NPs in water from up to120 min at $25{ }^{\circ} \mathrm{C}$. 


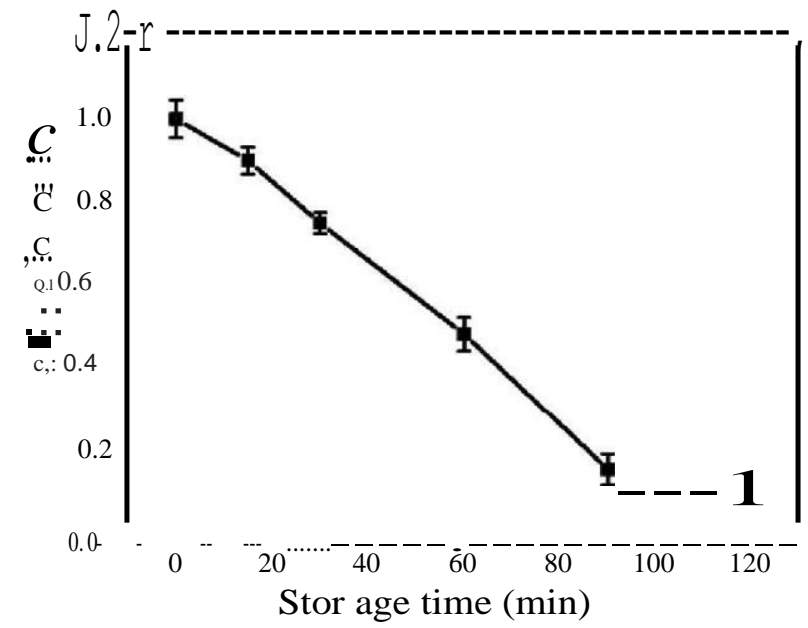

Figure S2 Degradation kinetics of free Cur at pH 5.5 PBS. 


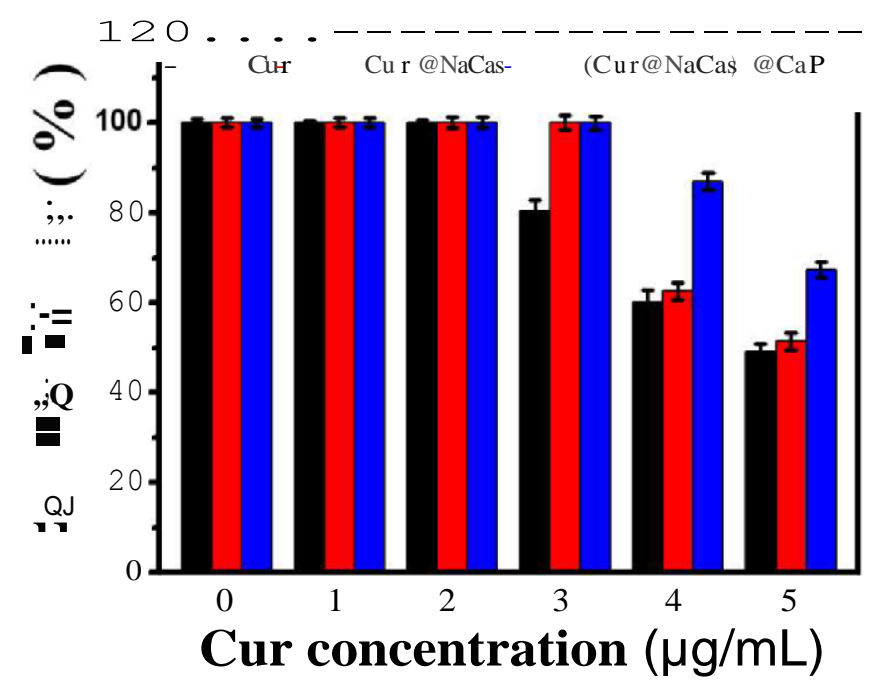

Figure S3 Anti-proliferation activity of 1, 2, 3, 4 and $5 \mu \mathrm{g} / \mathrm{mL}$ DMSO-dissolved and encapsulated Cur against MGC-803 cells, and treatment for $24 \mathrm{~h}$. 